\title{
Wickerhamomyces anomalus blood stream infection in a term newborn with pneumonia
}

\author{
Seda Yilmaz-Semerci ${ }^{1}$, Gamze Demirel$^{2}$, Ayhan Taştekin ${ }^{2}$ \\ ${ }^{1}$ Division of Neonatology, Department of Pediatrics, Istanbul Kanuni Sultan Süleyman Training and Research Hospital, \\ ${ }^{2}$ Division of Neonatology, Department of Pediatrics, İstanbul Medipol University, İstanbul, Turkey. \\ E-mail sedayilmazsemerci@gmail.com \\ Received: 28th November 2016, Revised: 4th February 2017, Accepted: 4th February 2017
}

\begin{abstract}
SUMMARY: Yılmaz-Semerci S, Demirel G, Taştekin A. Wickerhamomyces anomalus blood stream infection in a term newborn with pneumonia. Turk J Pediatr 2017; 59: 349-351.

The incidence of invasive candidiasis is high in neonates admitted to neonatal intensive care unit and is associated with significant morbidity and mortality rates. Candida albicans is the most common fungal agent pathogenic to neonates but invasive fungal infections caused by uncommon fungi have increased in recent years. Wickerhamomyces anomalus is a very rare pathogen causing blood stream infection in neonates, which has reportedly caused only few cases in the literature.
\end{abstract}

Here we report a case of blood stream infection caused by a fungal agent Wickerhamomyces anomalus in a term male infant.

Key words: wickerhamomyces anomalus, candidiasis, newborn.

The incidence of invasive candidiasis is high in neonates admitted to neonatal intensive care unit (NICU) and is associated with significant morbidity and mortality rates ${ }^{1}$. Candida albicans is the most common fungal agent pathogenic to neonates but invasive fungal infections caused by uncommon fungi have increased in recent years ${ }^{2,3}$. Wickerhamomyces anomalus (W. anomalus) is the teleomorph stages of several Candida species and is a very rare pathogen causing blood infection in neonates, which has reported caused only few cases of fungemia and a case of endocarditis in literature ${ }^{4}$. In this report we describe a case of an invasive $W$. anomalus infection in a term neonate in a tertiary NICU.

\section{Case Report}

A $3800 \mathrm{~g}$ term male newborn, at the 28th day of life, with the diagnosis of sepsis and pneumonia was admitted to the NICU. The participation of this case involved parental informed consents. He had had a cough, fever $\left(38-39.3{ }^{\circ} \mathrm{C}\right)$ and respiratory distress for three days. His laboratory results revealed white blood cell $49,900 / \mathrm{mm}^{3}$, hemoglobin $6.8 \mathrm{~g} / \mathrm{dl}$, hematocrit $20.7 \%$, platelets $1,084,000$, C-reactive protein
$271.87 \mathrm{mg} / \mathrm{dl}$, procalcitonin $4.03 \mathrm{ng} / \mathrm{ml}$. He required erythrocyte suspension transfusion once. Pediatric hematology department evaluated blood smear and leukocytosis and thrombocytosis, associated with infection, were noted. In spite of ampicillin and gentamycin therapy for seven days he had no clinical or laboratory recovery so his therapy was converted to teicoplanin and cefotaxime. He had both sonorous rhonchi and crepitant rales so salbutamol and budesonide nebules were added. Chest X-rays showed atelectasis of right upper lobe and bilateral diffuse infiltrations of the lungs (Fig. 1). After three days the neonates body temperature returned to normal. His first blood culture was reported as sterile. As the respiratory distress continued, the patient was consulted to the pediatric pulmonologist; high resolution computerized tomography (hRCT) was performed. HRCT showed us atelectasis and pneumonic infiltrations at left lung's inferior lobe and right lung's posterior segment of superior lobe (Fig. 2). At 9th day of the antibiotics, Candida pelliculosa (recognized as $W$. anomalus later) was isolated from blood cultures. As the isolated Candida was found to be sensitive to all - fluconazole, 
flucytosine, caspofungine, voriconazole and amphotericin, the patient was started on intravenous fluconazole therapy with a dose of $6 \mathrm{mg} / \mathrm{kg} /$ day. In the direction of clinical and laboratory recovery fluconazole therapy ended at the $14^{\text {th }}$ day. After 14 days of intravenous fluconazole, blood culture was negative. On the grounds of unexpected causative agent, pediatric immunology consultation was performed. Immunoglobulines were all normal and lower B lymphocyte accounts were thought to be related to overconsumption. The neonate was discharged from hospital in good health and was planned to be followed.

\section{Discussion}

Neonatal candidiasis has been reported to have crude mortality rates of $30 \%-60 \%$, and increases in rates with decreasing birth weight ${ }^{5,6}$. Although Candida albicans is the organism most often associated with serious fungal infections, other Candida species have emerged as clinically important pathogens associated with opportunistic infections ${ }^{7}$. When the literature was evaluated, it was seen that $W$. anomalus fungemia causes outbreaks in pediatric wards, while most of the adult cases were sporadic case reports ${ }^{8}$. W. anomalus is a yeast frequently found in various fruits, tree exudates, soil, vegetables and other organic compounds ${ }^{9}$. It has occasionally been reported

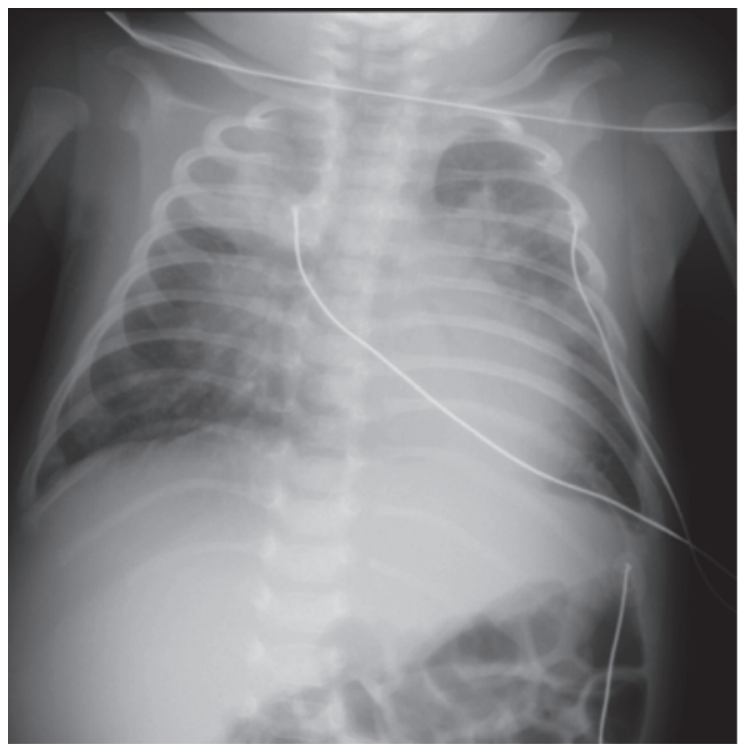

Fig. 1. Chest X-ray demonstrates atelectasis of right upper lobe and bilateral diffuse infiltrations of the lungs as a causative agent of fungaemia in both immunocompetent and immunocompromised patients, including those with AIDS ${ }^{10-20}$. W. anomalus has also been reported as a causative agent of nosocomial cerebral ventriculitis in low-birth-weight neonates, endocarditis in an intravenous drug abuser and urinary tract infection in a renal transplant recipient ${ }^{18}$, 21-23. Particularly, in the pediatric population $W$. anomalus is known to be responsible for serious nosocomial bloodstream infections of immunocompromised mostly as outbreaks in pediatric intensive care units ${ }^{8}$. In this report, we describe an invasive $W$. anomalus infection in a term neonate who was admitted because of pneumonia and respiratory distress. Our patient was given multiple courses of antibacterial antibiotics and parenteral nutrition for his severe enteral nutrition intolerance. However, he had no invasive operation or mechanical ventilation support. Before these symptoms, he had normal growth and nutrition. His hematologic and immunologic evaluation was completely normal. Risk factors were noted as prolonged NICU stay, prolonged parenteral nutrition and multiple antibiotic usages. Due to the patient's enteral feeding intolerance, gastroeosophageal reflux might thought to be a risk factor also. In a recent study carried out to analyze the antifungal susceptibility of clinical isolates belonging to seven uncommon species of Candida, of $15 \mathrm{~W}$. anomalus strains, eight were shown to be fluconazole resistant, six were itraconazole and ketoconazole resistant

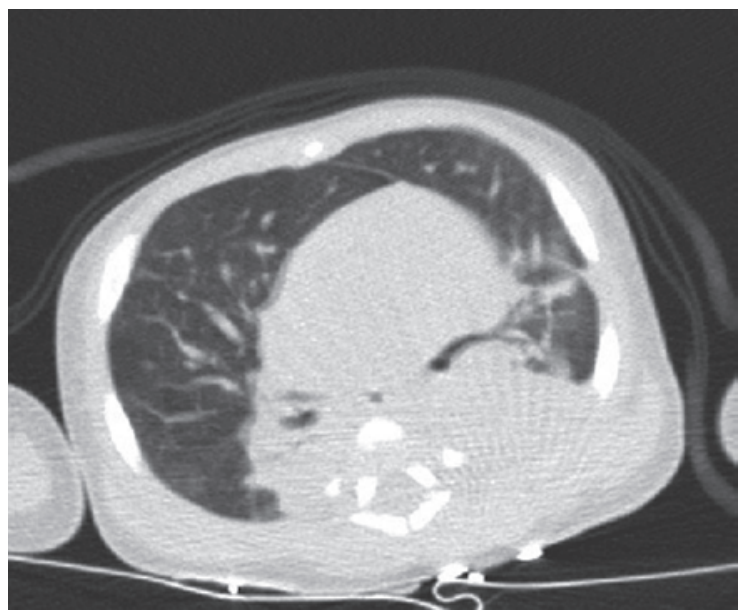

Fig. 2. HRCT shows atelectasis and pneumonic infiltrations at left lung's inferior lobe and right lung's posterior segment of superior lobe 
and one was flucytosine resistant ${ }^{24}$. This finding is particularly worrying, since, besides amphotericin $\mathrm{B}$, few therapeutic options are currently effective against this fungal infection. Fortunately, in our case, $W$. anomalus was treated successfully with fluconazole therapy for 14 days. Our patient further allows us to study W. anomalus and its ability to cause invasive infection. Since $W$. anomalus has not been included in widely used commercial yeast diagnostic kits (API 20C AUX, ID32C and Vitek-2), it is difficult for clinicians to properly identify and thus has been commonly misidentified as $P$. anomala or C. utilis only using commercial kit. In our patient, the pathogen was identified as $W$. anomalus by Vitek-2 kit. Identification and verification were provided by the hospital's clinical microbiology and infectious diseases laboratory.

As a conclusion, our case alerts us to be more suspicious about the fungal infections not only in preterm, also in term infants in NICU. An antifungal prophylaxis may be necessary for these patients when one or more risk factors exist. Non-albicans Candida species may rarely be an etiologic agent and they have not been included in widely used commercial yeast diagnostic kits. We must keep in mind these fungal pathogens as an infectious agent in neonates with risk factors.

\section{REFERENCES}

1. Zaoutis TE, Heydon K, Localio R, et al. Outcomes attributable to neonatal candidiasis. Clin Infect Dis 2007; 44: 1187-1193.

2. Bhally HS, Jain S, Shields C, et al. Infection in a neonate caused by Pichia fabianii: importance of molecular identification. Med Mycol 2006; 44: 185-187.

3. Gabriel F, Noel T, Accoceberry I. Lindnera (Pichia) fabianii blood infection after mesenteric ischemia. Med Mycol 2012; 50: 310-314.

4. Grenouillet F, Millon L, Chamouine A, et al. Pichia fabianii Fungemia in a Neonate. Pediatr Infect Dis J 2010; 29: 191.

5. Pappas PG, Rex JH, Lee J, et al. A prospective observational study of candidemia: epidemiology, therapy, and influences on mortality in hospitalized adult and pediatric patients. Clin Infect Dis 2003; 37 : 634-643.

6. Lopes JM, Goulart EM, Starling CE. Pediatric mortality due to nosocomial infection: a critical approach. Braz J Infect Dis 2007; 11: 515-519.

7. Hazen KC. New and emerging yeast pathogens. Clin Microbiol Rev 1995; 8: 462-478.
8. Kalkanci A, Dizbay M, Turan O, et al. Nosocomial transmission of Candida pelliculosa fungemia in a pediatric intensive care unit and review of the literature. Turk J Pediatr 2010; 52: 42-49.

9. Kwon-Chung KJ, Varma A, Edman JC, et al. Selection of ura5 and ura3 mutants from the two varieties of Cryptococcus neoformans on 5-fluoroorotic acid medium. J Med Vet Mycol 1992; 30: 61-69.

10. Alter SJ, Farley J. Development of Hansenula anomala infection in a child receiving fluconazole therapy. Pediatr Infect Dis J 1994; 13: 158-159.

11. Goss G, Grigg A, Rathbone P, et al. Hansenula anomala infection after bone marrow transplantation. Bone Marrow Transplant 1994; 14: 995-997.

12. Haron E, Anaissie E, Dumphy F, et al. Hansenula anomala fungemia. Rev Infect Dis 1988; 10: 1182-1186.

13. Hirasaki S, Ijichi T, Fujita N, et al. Fungemia caused by Hansenula anomala: successful treatment with fluconazole. Intern Med 1992; 31: 622-624.

14. Klein AS, Tortora GT, Malowitz R, et al. Hansenula anomala: a new fungal pathogen. Two case reports and a review of the literature. Arch Intern Med 1988; 148: 1210-1213.

15. Kunová A, Spánik S, Kollár T, et al. Breakthrough fungemia due to Hansenula anomala in a leukemic patient successfully treated with amphotericin B. Chemotherapy 1996; 42: 157-158.

16. Muñoz P, Garcia Leoni ME, Berenguer J, et al. Catheterrelated fungemia by Hansenula anomala. Arch Intern Med 1989; 149: 709-713.

17. Neumeister B, Rockemann M, Marre R. Fungaemia due to Candida pelliculosa in a case of acute pancreatitis. Mycoses 1992; 35: 309-310.

18. Salesa R, Burgos A, Fernandez-Mazarrasa C, et al. Transient fungaemia due to Candida pelliculosa in a patient with AIDS. Mycoses 1991; 34: 327-329.

19. Sekhon AS, Kowalewska-Grochowska K, Garg AK, et al. Hansenula anomala fungemia in an infant with gastric and cardiac complications with a review of the literature. Eur J Epidemiol 1992; 8: 305-308.

20. Taylor GD, Buchanan-Chell M, Kirkland T, et al. Trends and sources of nosocomial fungaemia. Mycoses 1994; 37: 187-190.

21. Murphy N, Buchanan CR, Damjanovic V, et al. Infection and colonisation of neonates by Hansenula anomala. Lancet 1986 8; 1: 291-293.

22. Nohinek B, Zee-Cheng CS, Barnes WG, et al. Infective endocarditis of a bicuspid aortic valve caused by Hansenula anomala. Am J Med 1987; 82: 165-168.

23. Qadri SM, Al Dayel F, Strampfer MJ, Cunha BA. Urinary tract infection caused by Hansenula anomala. Mycopathologia 1988; 104: 99-101.

24. Barchiesi F, Tortorano AM, Di Francesco LF, et al. In-vitro activity of five antifungal agents against uncommon clinical isolates of Candida spp. J Antimicrob Chemother 1999; 43: 295-299. 